\title{
Pulsed Sagnac polarization-entangled photon source with a PPKTP crystal at telecom wavelength
}

\author{
Rui-Bo Jin,,${ }^{1, *}$ Ryosuke Shimizu, ${ }^{2}$ Kentaro Wakui, ${ }^{1}$ Mikio Fujiwara, ${ }^{1}$ \\ Taro Yamashita, ${ }^{3}$ Shigehito Miki ${ }^{3}$ Hirotaka Terai, ${ }^{3}$ Zhen Wang, ${ }^{3,4}$ and \\ Masahide Sasaki ${ }^{1}$ \\ ${ }^{1}$ Advanced ICT Research Institute, National Institute of Information and Communications \\ Technology (NICT), 4-2-1 Nukui-Kitamachi, Koganei, Tokyo 184-8795, Japan \\ ${ }^{2}$ Center for Frontier Science and Engineering, University of Electro-Communications (UEC), \\ Tokyo 182-8585, Japan \\ ${ }^{3}$ Advanced ICT Research Institute, National Institute of Information and Communications \\ Technology (NICT), 588-2 Iwaoka, Kobe 651-2492, Japan \\ ${ }^{4}$ Shanghai Institute of Microsystem and Information Technology, Chinese Academy of \\ Sciences (CAS), 865 Changning Road, Shanghai 200050, China \\ *ruibo@nict.go.jp
}

\begin{abstract}
We demonstrate pulsed polarization-entangled photons generated from a periodically poled $\mathrm{KTiOPO}_{4}$ (PPKTP) crystal in a Sagnac interferometer configuration at telecom wavelength. Since the group-velocity-matching (GVM) condition is satisfied, the intrinsic spectral purity of the photons is much higher than in the previous scheme at around $800 \mathrm{~nm}$ wavelength. The combination of a Sagnac interferometer and the GVM-PPKTP crystal makes our entangled source compact, stable, highly entangled, spectrally pure and ultra-bright. The photons were detected by two superconducting nanowire single photon detectors (SNSPDs) with detection efficiencies of $70 \%$ and $68 \%$ at dark counts of less than $1 \mathrm{kcps}$. We achieved fidelities of $0.981 \pm 0.0002$ for $\left|\psi^{-}\right\rangle$and $0.980 \pm 0.001$ for $\left|\psi^{+}\right\rangle$respectively. This GVM-PPKTP-Sagnac scheme is directly applicable to quantum communication experiments at telecom wavelength, especially in free space.
\end{abstract}

(C) 2014 Optical Society of America

OCIS codes: (270.0270) Quantum optics; (190.4410) Nonlinear optics, parametric processes.

\section{References and links}

1. A. Poppe, A. Fedrizzi, R. Ursin, H. Böhm, T. Lorünser, O. Maurhardt, M. Peev, M. Suda, C. Kurtsiefer, H. Weinfurter, T. Jennewein, and A. Zeilinger, "Practical quantum key distribution with polarization entangled photons," Opt. Express 12, 3865-3871 (2004).

2. N. Gisin, S. Pironio, and N. Sangouard, "Proposal for implementing device-independent quantum key distribution based on a heralded qubit amplifier," Phys. Rev. Lett. 105, 070501 (2010).

3. J. Yin, J.-G. Ren, H. Lu, Y. Cao, H.-L. Yong, Y.-P. Wu, C. Liu, S.-K. Liao, F. Zhou, Y. Jiang, X.-D. Cai, P. Xu, G.S. Pan, J.-J. Jia, Y.-M. Huang, H. Yin, J.-Y. Wang, Y.-A. Chen, C.-Z. Peng, and J.-W. Pan, "Quantum teleportation and entanglement distribution over 100-kilometre free-space channels," Nature 488, 185-188 (2012).

4. P. Kok, W. J. Munro, K. Nemoto, T. C. Ralph, J. P. Dowling, and G. J. Milburn, "Linear optical quantum computing with photonic qubits," Rev. Mod. Phys. 79, 135-174 (2007).

5. K. Edamatsu, "Entangled photons: generation, observation, and characterization," Jpn. J. Appl. Phys. 46, 71757187 (2007). 
6. P. G. Kwiat, K. Mattle, H. Weinfurter, A. Zeilinger, A. V. Sergienko, and Y. Shih, "New high-intensity source of polarization-entangled photon pairs," Phys. Rev. Lett. 75, 4337-4341 (1995).

7. B.-S. Shi and A. Tomita, "Generation of a pulsed polarization entangled photon pair using a Sagnac interferometer," Phys. Rev. A 69, 013803 (2004).

8. J. Altepeter, E. Jeffrey, and P. Kwiat, "Phase-compensated ultra-bright source of entangled photons," Opt. Express 13, 8951-8959 (2005).

9. Y. Li, H. Jing, and M.-S. Zhan, "Optical generation of a hybrid entangled state via an entangling single-photonadded coherent state," J. Phys. B: At. Mol. Opt. Phys. 39, 2107-2113 (2006).

10. M. Hentschel, H. Hübel, A. Poppe, and A. Zeilinger, "Three-color Sagnac source of polarization-entangled photon pairs," Opt. Express 17, 23153-23159 (2009).

11. R.-B. Jin, R. Shimizu, F. Kaneda, Y. Mitsumori, H. Kosaka, and K. Edamatsu, "Entangled-state generation with an intrinsically pure single-photon source and a weak coherent source," Phys. Rev. A 88, 012324 (2013).

12. F. Steinlechner, S. Ramelow, M. Jofre, M. Gilaberte, T. Jennewein, J. P. Torres, M. W. Mitchell, and V. Pruneri, "Phase-stable source of polarization-entangled photons in a linear double-pass configuration," Opt. Express 21, 11943-11951 (2013).

13. T. Kim, M. Fiorentino, and F. N. C. Wong, "Phase-stable source of polarization-entangled photons using a polarization Sagnac interferometer," Phys. Rev. A 73, 012316 (2006).

14. F. Wong, J. Shapiro, and T. Kim, "Efficient generation of polarization-entangled photons in a nonlinear crystal," Laser Phys. 16, 1517-1524 (2006).

15. A. Fedrizzi, T. Herbst, A. Poppe, T. Jennewein, and A. Zeilinger, "A wavelength-tunable fiber-coupled source of narrowband entangled photons," Opt. Express 15, 15377-15386 (2007).

16. O. Kuzucu and F. N. C. Wong, "Pulsed Sagnac source of narrow-band polarization-entangled photons," Phys. Rev. A 77, 032314 (2008).

17. A. Predojević, S. Grabher, and G. Weihs, "Pulsed Sagnac source of polarization entangled photon pairs," Opt. Express 20, 25022-25029 (2012).

18. A. Fedrizzi, R. Ursin, T. Herbst, M. Nespoli, R. Prevedel, T. Scheidl, F. Tiefenbacher, T. Jennewein, and A. Zeilinger, "High-fidelity transmission of entanglement over a high-loss free-space channel," Nat. Phys. 5, 389-392 (2009).

19. R. Prevedel, D. R. Hamel, R. Colbeck, K. Fisher, and K. J. Resch, "Experimental investigation of the uncertainty principle in the presence of quantum memory and its application to witnessing entanglement," Nat. Phys. 7, 757-761 (2011).

20. L. Vermeyden, M. Bonsma, C. Noel, J. M. Donohue, E. Wolfe, and K. J. Resch, "Experimental violation of three families of Bell's inequalities," Phys. Rev. A 87, 032105 (2013).

21. S. Ramelow, A. Mech, M. Giustina, S. Gröblacher, W. Wieczorek, J. Beyer, A. Lita, B. Calkins, T. Gerrits, S. W. Nam, A. Zeilinger, and R. Ursin, "Highly efficient heralding of entangled single photons," Opt. Express 21, 6707-6717 (2013).

22. M. Giustina, A. Mech, S. Ramelow, B. Wittmann, J. Kofler, J. Beyer, A. Lita, B. Calkins, T. Gerrits, S. W. Nam, R. Ursin, and A. Zeilinger, "Bell violation using entangled photons without the fair-sampling assumption," Nature 497, 227-230 (2013).

23. Y. Cao, H. Liang, J. Yin, H.-L. Yong, F. Zhou, Y.-P. Wu, J.-G. Ren, Y.-H. Li, G.-S. Pan, T. Yang, X. Ma, C.-Z. Peng, and J.-W. Pan, "Entanglement-based quantum key distribution with biased basis choice via free space," Opt. Express 21, 27260-27268 (2013).

24. I. A. Grice, W. P. \& Walmsley, "Spectral information and distinguishability in type-II down-conversion with a broadband pump," Phys. Rev. A 56, 1627-1634 (1997).

25. T. E. Keller and M. H. Rubin, "Theory of two-photon entanglement for spontaneous parametric down-conversion driven by a narrow pump pulse," Phys. Rev. A 56, 1534-1541 (1997).

26. F. König and F. N. C. Wong, "Extended phase matching of second-harmonic generation in periodically poled $\mathrm{KTiOPO}_{4}$ with zero group-velocity mismatch," Appl. Phys. Lett. 84, 1644 (2004).

27. P. J. Mosley, J. S. Lundeen, B. J. Smith, P. Wasylczyk, A. B. U'Ren, C. Silberhorn, and I. A. Walmsley, "Heralded generation of ultrafast single photons in pure quantum states," Phys. Rev. Lett. 100, 133601 (2008).

28. R.-B. Jin, J. Zhang, S. Ryosuke, M. Nobuyuki, M. Yasuyoshi, K. Hideo, and E. Keiichi, "High-visibility nonclassical interference between intrinsically pure heralded single photons and photons from a weak coherent field," Phys. Rev. A 83, 031805 (2011).

29. R.-B. Jin, R. Shimizu, K. Wakui, H. Benichi, and M. Sasaki, "Widely tunable single photon source with high purity at telecom wavelength," Opt. Express 21, 10659-10666 (2013).

30. P. G. Evans, R. S. Bennink, W. P. Grice, T. S. Humble, and J. Schaake, "Bright source of spectrally uncorrelated polarization-entangled photons with nearly single-mode emission," Phys. Rev. Lett. 105, 253601 (2010).

31. A. Eckstein, A. Christ, P. J. Mosley, and C. Silberhorn, "Highly efficient single-pass source of pulsed single-mode twin beams of light," Phys. Rev. Lett. 106, 013603 (2011).

32. T. Lutz, P. Kolenderski, and T. Jennewein, "Toward a downconversion source of positively spectrally correlated and decorrelated telecom photon pairs," Opt. Lett. 38, 697-699 (2013).

33. T. Lutz, P. Kolenderski, and T. Jennewein, "Demonstration of spectral correlation control in a source of polariza- 
tion entangled photon pairs at telecom wavelength," arXiv:1309.4040 (2013).

34. B. J. Smith, P. Mahou, O. Cohen, J. S. Lundeen, and I. A. Walmsley, "Photon pair generation in birefringent optical fibers," Opt. Express 17, 23589-23602 (2009).

35. T. Gerrits, M. J. Stevens, B. Baek, B. Calkins, A. Lita, S. Glancy, E. Knill, S. W. Nam, R. P. Mirin, R. H. Hadfield, R. S. Bennink, W. P. Grice, S. Dorenbos, T. Zijlstra, T. Klapwijk, and V. Zwiller, "Generation of degenerate, factorizable, pulsed squeezed light at telecom wavelengths," Opt. Express 19, 24434-24447 (2011).

36. R.-B. Jin, K. Wakui, R. Shimizu, H. Benichi, S. Miki, T. Yamashita, H. Terai, Z. Wang, M. Fujiwara, and M. Sasaki, "Nonclassical interference between independent intrinsically pure single photons at telecommunication wavelength," Phys. Rev. A 87, 063801 (2013).

37. N. Bruno, A. Martin, T. Guerreiro, B. Sanguinetti, and R. T. Thew, "Pulsed source of spectrally uncorrelated and indistinguishable photons at telecom wavelengths," arXiv:1403.6740 (2014).

38. T. Y. Fan, C. E. Huang, B. Q. Hu, R. C. Eckardt, Y. X. Fan, R. L. Byer, and R. S. Feigelson, "Second harmonic generation and accurate index of refraction measurements in flux-grown $\mathrm{KTiOPO}_{4}$," Appl. Opt. 26, 2390-2394 (1987).

39. K. Fradkin, A. Arie, A. Skliar, and G. Rosenman, "Tunable midinfrared source by difference frequency generation in bulk periodically poled $\mathrm{KTiOPO}_{4}$," Appl. Phys. Lett. 74, 914-916 (1999).

40. A. Scherer, B. C. Sanders, and W. Tittel, "Long-distance practical quantum key distribution by entanglement swapping," Opt. Express 19, 3004-3018 (2011).

41. Y.-F. Huang, B.-H. Liu, L. Peng, Y.-H. Li, L. Li, C.-F. Li, and G.-C. Guo, "Experimental generation of an eightphoton Greenberger-Horne-Zeilinger state," Nat. Commun. 2, 546(1-6) (2011).

42. X.-C. Yao, T.-X. Wang, P. Xu, H. Lu, G.-S. Pan, X.-H. Bao, C.-Z. Peng, C.-Y. Lu, Y.-A. Chen, and J.-W. Pan, "Observation of eight-photon entanglement," Nat. Photon. 6, 225-228 (2012).

43. J.-W. Pan, Z.-B. Chen, L. Chao-Yang, H. Weinfurter, A. Zeilinger, and Żukowski Marek, "Multiphoton entanglement and interferometry," Rev. Mod. Phys. 84, 777-838 (2012).

44. http://demonstrations.wolfram.com/sagnacinterferometer/

45. S. Miki, T. Yamashita, T. Hirotaka, and W. Zhen, "High performance fiber-coupled nbtin superconducting nanowire single photon detectors with Gifford-Mcmahon cryocooler,' Opt. Express 21, 10208-10214 (2013).

46. T. Yamashita, S. Miki, H. Terai, and Z. Wang, "Low-filling-factor superconducting single photon detector with high system detection efficiency," Opt. Express 21, 27177-27184 (2013).

47. S. Miki, M. Fujiwara, M. Sasaki, and Z. Wang, "NbN superconducting single-photon detectors prepared on single-crystal MgO substrates," IEEE Trans. Appl. Superconduct. 17, 285-288 (2007).

48. R.-B. Jin, M. Fujiwara, T. Yamashita, S. Miki, H. Terai, Z. Wang, K. Wakui, R. Shimizu, and M. Sasaki, "Efficient detection of a highly bright photon source using superconducting nanowire single photon detectors," arXiv:1309.1221 (2013).

49. D. R. Hamel, "Realization of novel entangled photon sources using periodically poled materials," Master's thesis, University of Waterloo (2010).

50. J. F. Clauser and A. Shimony, "Bell's theorem : experimental tests and implications," Rep. Prog. Phys. 41, 1881 (1978).

51. J. F. Clauser, M. A. Horne, A. Shimony, and R. A. Holt, "Proposed experiment to test local hidden-variable theories," Phys. Rev. Lett. 23, 880-884 (1969).

52. D. F. V. James, P. G. Kwiat, W. J. Munro, and A. G. White, "Measurement of qubits," Phys. Rev. A 64, 052312 (2001).

53. R. Jozsa, "Fidelity for mixed quantum states," J. Mod. Opt. 41, 2315-2323 (1994).

54. W. K. Wootters, "Entanglement of formation of an arbitrary state of two qubits," Phys. Rev. Lett. 80, 2245-2248 (1998).

55. I. Morohashi, T. Sakamoto, H. Sotobayashi, T. Kawanishi, I. Hosako, and M. Tsuchiya, "Widely repetitiontunable 200 fs pulse source using a Mach-Zehnder-modulator-based flat comb generator and dispersion-flattened dispersion-decreasing fiber," Opt. Lett. 33, 1192-1194 (2008).

56. J. T. Barreiro, N. K. Langford, N. A. Peters, and P. G. Kwiat, "Generation of hyperentangled photon pairs," Phys. Rev. Lett. 95, 260501 (2005).

\section{Introduction}

Polarization is an important degree of freedom for photons. Polarization-entangled photons are fundamental quantum resources in quantum information processing for many applications, such as quantum key distribution [1], photon amplifiers [2], quantum teleportation [3] and quantum computation [4]. The widely used technique for generating the polarization-entangled state is based on a spontaneous parametric downconversion (SPDC) process, which can be arranged in various configurations and with different crystals [5-12]. Recently, polarization-entangled photons from a periodically poled $\mathrm{KTiOPO}_{4}$ (PPKTP) crystal in a Sagnac interferometer con- 
figuration has become a hot topic, since this source has the merits of compactness, stability and high brightness. Kim et al, demonstrated the first entangled photon source with a PPKTP crystal in a Sagnac-loop with a continuous-wave (cw) pump laser at $405 \mathrm{~nm}$ in 2006 [13, 14]. Then, Fedrizzi et al, presented an optimized scheme with a cw pump laser in 2007 [15]. However, the cw pumped SPDC source cannot provide any timing information about when the photon pair is generated, which is important for applications such as synchronization with system clocks in quantum communication systems. Therefore, Kuzucu and Wong developed a pulsed Sagnac polarization-entangled source at $780 \mathrm{~nm}$ in 2008 [16]. In 2012, Predojević et $a l$, investigated the phase property of this system [17]. Now this PPKTP-Sagnac scheme has become a common tool for the generation of polarization-entangled state and has applied in many experiments [18-23].

However, in all the previous experiments [13-23], the entangled photons were generated at around $800 \mathrm{~nm}$ wavelength. In this work, we expand such pulsed PPKTP-Sagnac scheme into a group-velocity matched (GVM) regime and demonstrate a GVM-PPKTP-Sagnac scheme at the telecom wavelength. The concept of GVM in SPDC was introduced by Grice and Walmsley [24], and by Keller and Rubin [25] in 1997. PPKTP crystal with GVM condition was first experimentally investigated by König and Wong [26] for second-harmonic generation in 2004. The GVM condition in SPDC has been experimentally realized in KDP crystal at $830 \mathrm{~nm}$ wavelength [27, 28], PPKTP crystal at around $1584 \mathrm{~nm}$ wavelength [29,-31], and BBO crystal at $1550 \mathrm{~nm}$ wavelength [32,33]. The GVM condition in spontaneous four-wave mixing has also been demonstrated in optical fibers [34]. In the case of PPKTP crystal, the GVM condition means $2 V_{g, p}^{-1}=V_{g, s}^{-1}+V_{g, i}^{-1}$, where $V_{g, \mu}^{-1}(\mu=p, s, i)$ is the inverse of the group velocity $V_{g, \mu}$ for the pump $p$, the signal $s$, and the idler $i$.

With the GVM condition, the PPKTP crystal may have a high spectral purity at telecom wavelengths [29]. The spectral purity, a parameter describing the degree of spectral uncorrelation between the signal and idler photons, is defined as $p=\operatorname{Tr}\left(\hat{\rho}_{s}^{2}\right)=\operatorname{Tr}\left(\hat{\rho}_{i}^{2}\right)$, where $\hat{\rho}_{s(i)}$ is the reduced density operator of the signal $(s)$ or idler $(i)$, and $\operatorname{Tr}$ represents the partial trace. The spectral purity is calculated by applying Schmidt decomposition on the join spectral amplitude of the signal and idler photons. See [29] for more details about spectral purity. See [30, 31, 35-37] for the experimentally measured joint spectral intensities of the GVMPPKTP crystal by several different groups. Here, we compare the maximal intrinsic spectral purity of PPKTP crystal at around $800 \mathrm{~nm}$ and $1550 \mathrm{~nm}$ in Fig. 11. The maximal intrinsic spectral purity of 0.16 at the $800 \mathrm{~nm}$ range is much lower than that at the $1550 \mathrm{~nm}$ range, 0.82 .

Spectral purity is of paramount importance for experiments with multi-entangled-source. For example, in the entanglement swapping [40] or multi-photon entangled state generation experiments [41, 42], the spectral purity of each source must be highly pure to achieve high interference visibilities [43]. At $800 \mathrm{~nm}$, to improve the purity from 0.16 to unity, we need to adopt very narrow bandpass filters to improve the purity, and the brightness will be largely decreased. However, only coarse bandpass filters can improve the purity from 0.82 to near unity at telecom wavelength range [29]. Therefore, in principle, the GVM-PPKTP-Sagnac photon source at telecom wavelengths might be much brighter than the PPKTP-Sagnac scheme at the $800 \mathrm{~nm}$ range for multi-entangled-source applications.

Besides the high spectral purity, another important merit of our GVM-PPKTP-Sagnac scheme is that such high-quality polarization-entangled photons at telecom wavelengths are suitable for long-haul transmission using low-loss optical fibers. This establishes the basis for many quantum info-communication applications at telecom wavelength.

Furthermore, low-efficiency photon detectors were obstacles for the telecom-band experiments, but the rapid development in superconducting nanowire single photon detector (SNSPD) 

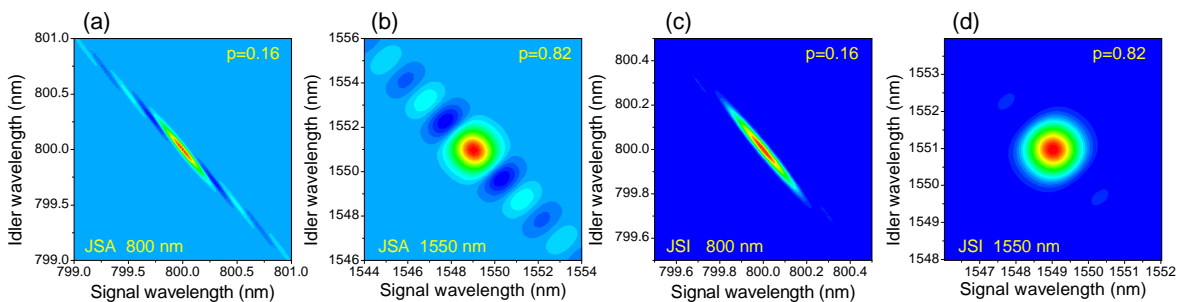

Fig. 1. Typical joint spectral amplitude (JSA, a, b) and joint spectral intensity (JSI, c, d) of the down-converted photons from a PPKTP crystal at $800 \mathrm{~nm}(\mathrm{a}, \mathrm{c})$ and $1550 \mathrm{~nm}$ (b, d), with corresponding maximal spectral purities (p) of 0.16 and 0.82 , respectively. In this simulation, we fixed the crystal lengths at $30 \mathrm{~mm}$, and scanned the full width at half maximum (FWHM) of the pump so as to obtain the maximal purities. For (a, c), with a pump laser at $400 \mathrm{~nm}$, the maximal purity was achieved at 0.16 with an FWHM of 0.014 $\mathrm{nm}(16.8 \mathrm{ps})$, and for (b, d) with a pump laser at $775 \mathrm{~nm}$, the maximal purity was 0.82 with an FWHM of $0.4 \mathrm{~nm}(2.3 \mathrm{ps})$. (a, c) were calculated with the Sellmeier equations from [38] for y direction and [39] for $\mathrm{z}$ direction. $(\mathrm{b}, \mathrm{d})$ were calculated with the Sellmeier equations from [26] for y direction and [39] for $\mathrm{z}$ direction. The spectra of the signal and idler photons in (b, d) have a Gaussian shape with a bandwidth of around $1.2 \mathrm{~nm}$. See [29] for more details of the simulations $(b, d)$.

technologies has overcome this disadvantage. In this paper, we demonstrate an ultra-bright polarization entangled photon source with high spectral purity, and detected by state-of-the-art SNSPDs developed by our group.

\section{Experiment}

The experimental setup is shown in Fig. 2. In this Sagnac interferometer configuration, the pump beam is split into two, the clockwise (CW) pump and the counterclockwise (CCW) pump. Both the CW and CCW pump beams are in opposite directions, but follow the same path, therefore this scheme is robust against the optical path changes and can keep phase ultra stable. Another important feature of this configuration is that the temporal walk-off between the signal (with higher group velocity) and idler (with lower group velocity) can be automatically cancelled out, since the signal (idler) generated by $\mathrm{CW}$ pump propagates along with the signal (idler) generated by the CCW pump. A fine alignment of the Sagnac loop is not easy. Therefore, we make a mathematical simulation [44] to simulate the beam propagations in a triangle shape Sagnac-loop. From this simulation, we can learn that the output beams are always in parallel, but never cross. In order to achieve a completely collinear configuration for both the $\mathrm{CW}$ and CCW pump beams, the residual pump beams must overlap with the input laser.

Our superconducting nanowire single photon detectors (SNSPDs) are fabricated with 5-9 nm thick and $80-100 \mathrm{~nm}$ wide niobium nitride $(\mathrm{NbN})$ or niobium titanium nitride $(\mathrm{NbTiN})$ meander nanowires on thermally oxidized silicon substrates [45 46]. The nanowire covers an area of 15 $\mu \mathrm{m} \times 15 \mu \mathrm{m}$. The SNSPDs are installed in a Gifford-McMahon cryocooler system and are cooled to 2.1 Kelvin. The maximum system detection efficiency (SDE) is $79 \%$ with a dark count rate (DCR) of $2 \mathrm{kcps}$. The measured timing jitter and dead time (recovery time) were $68 \mathrm{ps}[45]$ and $40 \mathrm{~ns}$ [47]. In this experiment, the SDEs of the two SNSPDs were set at $70 \%$ and $68 \%$, corresponding to DCRs of less than $1 \mathrm{kcps}$. In our previous experiment, we achieved coincidence counts of $400 \mathrm{kcps}(1.17 \mathrm{Mcps})$ at a pump power of $100 \mathrm{~mW}(400 \mathrm{~mW})$ with our PPKTP crystal and SNSPDs [48]. 


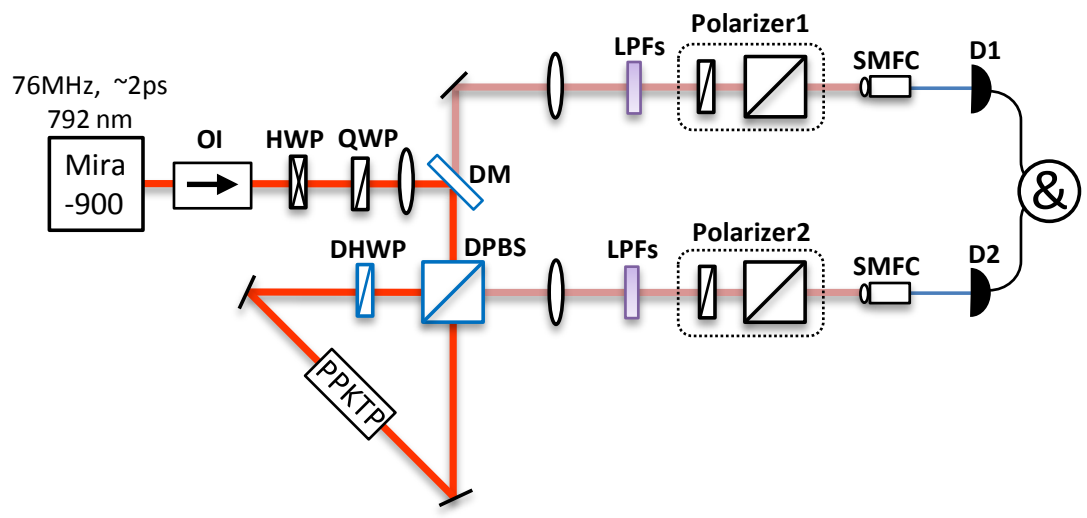

Fig. 2. The experimental setup. Picosecond laser pulses ( $76 \mathrm{MHz}, 792 \mathrm{~nm}$, temporal duration $\sim 2$ ps) from a mode-locked Titanium sapphire laser (Mira900, Coherent Inc.) passed through an optical isolator (OI), a half-wave plate (HWP) and a quarter-wave plate (QWP). Then the pulses were focused by a $f=200 \mathrm{~mm}$ lens (beam waist $\sim 45 \mu \mathrm{m}$ ), reflected by a dichroic mirror (DM: DMLP1180, Thorlabs) and guided into a Sagnac-loop. The Sagnac-loop consisted of a dual-wavelength polarization beam splitter (DPBS, extinction ratio $=200: 1$, Union Optics), a dual-wavelength HWP (DHWP, for both $792 \mathrm{~nm}$ and $1584 \mathrm{~nm}$, Union Optics), and a 30-mm-long PPKTP crystal with a polling period of 46.1 $\mu \mathrm{m}$ for a type-II collinear group-velocity-matched SPDC. The temperature of the PPKTP was maintained at $32.5^{\circ} \mathrm{C}$ to achieve a degenerate wavelength at $1584 \mathrm{~nm}$. The PPKTP crystal was pumped by clockwise $(\mathrm{CW})$ and counterclockwise $(\mathrm{CCW})$ laser pulses at the same time. The DHWP is set at 45 degree to make the CCW pump horizontally polarized. The down-converted photons, i.e., the signal and idler, were collimated by another two $f=200 \mathrm{~mm}$ lenses, filtered by longpass filters (LPFs) and then coupled into singlemode fibers by two couplers (SMFC). Finally, all the collected photons were sent to two superconducting nanowire single-photon detectors (SNSPDs), which were connected to a coincidence counter (\&). To test the polarization correlation, we inserted two sets of Polarizers (HWP+PBS) before SMFCs. To carry out quantum state tomography, we replaced the combination of HWP+PBS with that of HWP+QWP+PBS. Since the SNSPDs were polarization dependent, the photons input into the SNSPD were adjusted by fiber-polarization controllers (not shown). The overall efficiency was estimated as 0.10 , including the detectors' average efficiency of 0.69 , the SMFCs' average collection efficiency of 0.23 and the whole optics' transmission efficiency of 0.64 . 
(a)

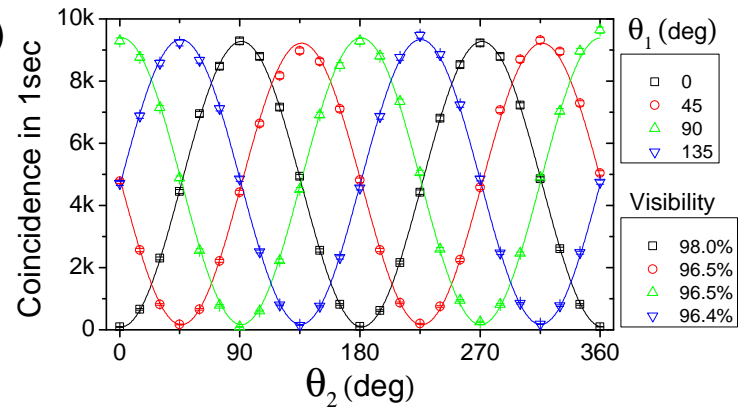

(b)

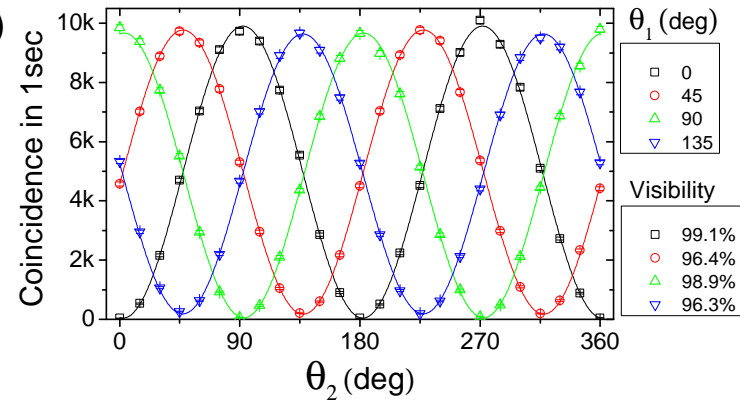

Fig. 3. Two-fold coincidence counts in one second as a function of the two polarizers, with a pump power of $10 \mathrm{~mW}$. (a) for $\left|\psi^{-}\right\rangle$state, (b) for $\left|\psi^{+}\right\rangle$state. The background counts have been subtracted. The error bars were added by assuming Poissonian statistics of these coincidence counts.

The output state of this scheme is

$$
|\Psi\rangle \propto|H\rangle|V\rangle+e^{i \phi} \beta|V\rangle|H\rangle,
$$

where $\phi$ is the relative phase between the two paths in the CW and CCW directions, and $\beta$ is the ratio of the two pumps [13]. By rotating the angle of QWP and HWP, we change $\phi$ and $\beta$. By slightly moving the position of PPKTP, we can finely adjust the relative phase $\phi$ by changing the Gouy phase [17. 49]. We can easily exchange the state between $\left|\psi^{-}\right\rangle=\frac{1}{\sqrt{2}}(|H V\rangle-|V H\rangle)$ and $\left|\psi^{+}\right\rangle=\frac{1}{\sqrt{2}}(|H V\rangle+|V H\rangle)$ by rotating the angle of QWP and HWP.

We set the pump power at $10 \mathrm{~mW}$ and carried out a polarization correlation measurement by recording the coincidence counts while changing the angles $\theta_{1}$ and $\theta_{2}$ of Polarizer 1 and Polarizer 2, respectively. The experimental results for some fixed values of $\theta_{1}\left(\theta_{1}=0,45,90\right.$ and 135 degrees) are shown in Fig. 3. For $\left|\psi^{-}\right\rangle$state in Fig. 3.a), after background subtraction, the visibilities were $98.0 \%, 96.5 \%, 96.5 \%, 96.4 \%$ for Polarizer 1 at $0,45,90$ and 135 degrees, respectively. Before background subtraction, the visibilities were 96.7\%, 95.3\%, 95.5\% and $95.2 \%$, respectively. For $\left|\psi^{+}\right\rangle$state in Fig. 3 b), the background subtracted visibilities were $99.1 \%, 96.4 \%, 98.9 \%, 96.3 \%$ for Polarizer 1 equaled to $0,45,90$ and 135 degree, respectively. Before background subtraction, the visibilities were $97.4 \%, 95.2 \%, 98.0 \%$ and $95.0 \%$. Besides the background counts, other two reasons for the degradation of the visibilities were the imperfect compensation of the phase $\phi$ in Eq.(1), and the low extinction ratio of the DPBS (around 200:1). The measured maximum coincidence count in Fig. 3 was $10 \mathrm{kcps}$, which corresponded to a coincidence of $20 \mathrm{kcps}$ without polarizers.

All the fringe visibilities in Fig. 3 were higher than $96 \%$, which exceeded $71 \%$, the bound required to violate the Bell's inequality (also called Bell-CHSH inequality) [50]. We measured 

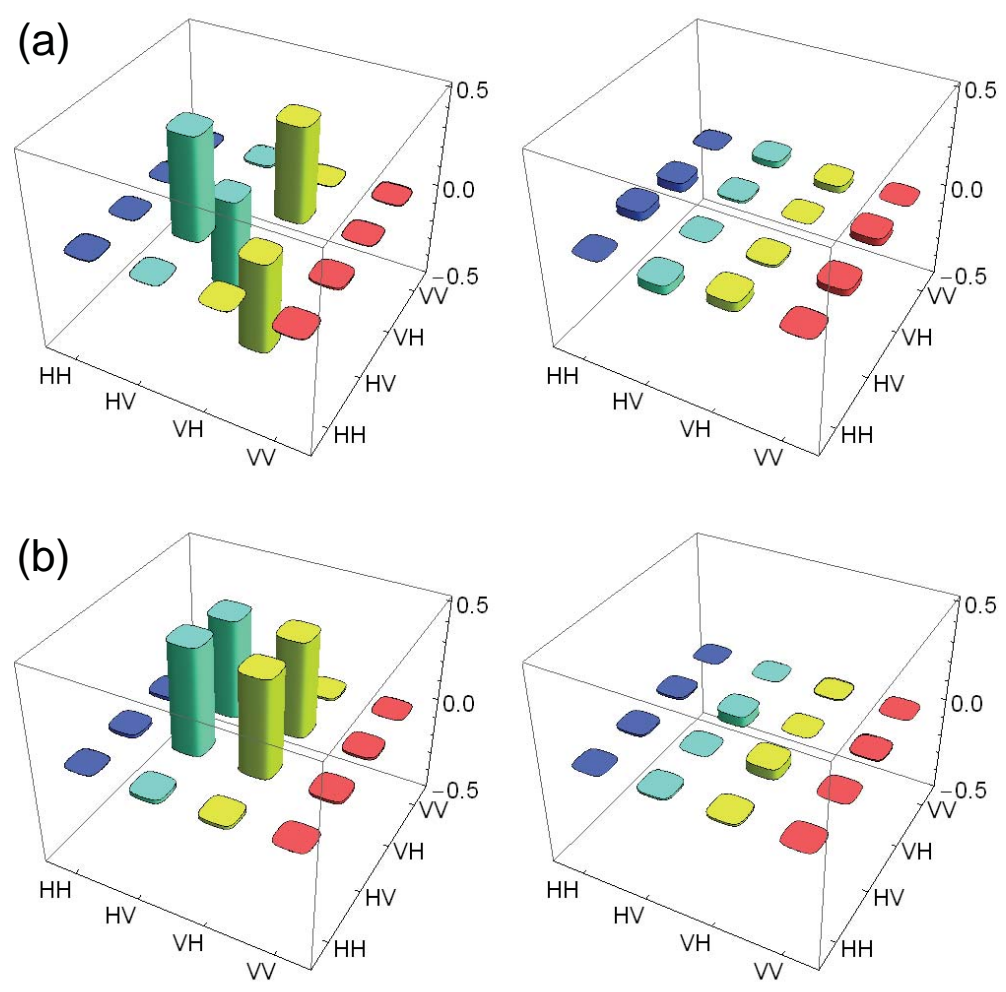

Fig. 4. Real (left) and imaginary (right) parts of the reconstructed density matrix. (a) for $\left|\psi^{-}\right\rangle$state, (b) for $\left|\psi^{+}\right\rangle$state.

the Bell parameter $S$, which directly indicated the violation of Bell's inequality [51]. For $\left|\psi^{-}\right\rangle$ state, the obtained value of $S$ was $2.75 \pm 0.01(75 \sigma)$ with $1 \mathrm{~s}$ accumulation time for each polarizer set, and $2.75 \pm 0.003(250 \sigma)$ with $10 \mathrm{~s}$. Without background subtraction, the raw $S$ was $2.72 \pm 0.01$ for $1 \mathrm{~s}$, and $2.72 \pm 0.003$ for $10 \mathrm{~s}$. For $\left|\psi^{+}\right\rangle$state, the obtained value of $S$ was $2.76 \pm 0.01(76 \sigma)$ for $1 \mathrm{~s}$ accumulation time for each polarizer set, $2.75 \pm 0.003(250 \sigma)$ for $10 \mathrm{~s}$, and $2.76 \pm 0.001(760 \sigma)$ for $100 \mathrm{~s}$.

We also carried out state tomography of our two-photon polarization state. Polarizer 1 and 2 in Fig. 2 were replaced by combinations of HWP, QWP and PBS, to allow polarization correlation analysis in not only linear but also circular polarization bases. The density matrix $\rho_{\text {exp }}$ reconstructed with a maximum likelihood estimation method [52] is shown in Fig. 4 The fidelities [53], $F \equiv\left\langle\psi^{ \pm}\left|\rho_{\exp }\right| \psi^{ \pm}\right\rangle$, to the ideal Bell state $\left|\psi^{ \pm}\right\rangle$, were estimated as $0.981 \pm 0.0002$ $(0.973 \pm 0.0002)$ for $\left|\psi^{-}\right\rangle$and $0.980 \pm 0.001(0.968 \pm 0.001)$ for $\left|\psi^{+}\right\rangle$with background subtracted data (raw data) accumulated in 10s. The corresponding concurrences [54] were 0.981 $\pm 0.0004(0.971 \pm 0.0008)$ and $0.969 \pm 0.002(0.956 \pm 0.002)$, respectively. These values indicated that our states were highly entangled.

To investigate the effect of multi-pair emission on the entangled state, we measured the visibility as a function of pump power, as shown in Fig. 5. The raw visibilities exhibited a linear decrease with respect to the increase of the pump power, providing evidence of multi-pair generation at higher pump power [30]. After background subtraction, the visibility was almost fixed at $96 \%$ for pump power from $10 \mathrm{~mW}$ to $100 \mathrm{~mW}$. In the future, this low visibility at high pump power can be improved by using a laser with high repetition rate and low average power per pulse, such as the $10 \mathrm{GHz}$-repetition-rate comb laser [55]. 


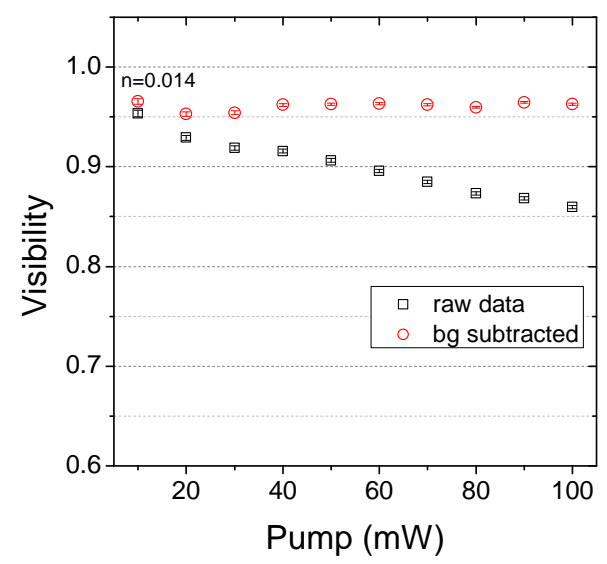

Fig. 5. Raw and background subtracted visibilities with Polarizer 1 set at 45 degrees for the $\left|\psi^{-}\right\rangle$state as a function of incident pump power. The uncertainties of these visibilities were derived using Poissonian errors on the coincidence counts. The left two points corresponds to the data in Fig. 3 and 4, with an average photon numbers per pulse of 0.014 .

\section{Discussion and Outlook}

Comparing our scheme with the previous entangled photon source with a GVM-PPKTP crystal in a calcite beam displacer configuration at telecom wavelength [30], our count rates are more than 100 times higher, mainly thanks to our highly efficient SNSPDs and fine alignment in Sagnac-loop. Obtaining entangled photon pairs with a high count rate and a low pump power at telecom wavelength is an important feature of our scheme.

In this experiment, we set the wavelength of the signal and the idler to be the same. By changing the temperature, we could also obtain non-degenerated entangled photons, which can be used to prepare a frequency-entangled state or hyper-entangled state at telecom wavelengths [56]. Since the PPKTP crystal has the property of a spectrally wide tunablility with high purity [29], the source can also be a wavelength-widely-tunable entangled photon source by using broadband dual-wavelength HWP and PBS in Fig.2.

In the future, this polarization-entangled photon source will be useful for a variety of applications in quantum information and communication at telecom wavelengths. For example, this source is directly applicable to free space quantum key distribution at telecom wavelengths and short distance fiber communications. It can also be applied to quantum communication experiments using multiple entangled photon sources, such as quantum teleportation and entanglement swapping. By changing this polarization-entangled photon source to a time-bin entangled photon source by simply using a Mach-Zehnder-type delay system, this source can be used for a long-distance fiber-based quantum key distribution system.

\section{Conclusion}

In summary, we have demonstrated a polarization-entangled photon source with a PPKTP crystal in a Sagnac interferometer configuration. The PPKTP crystal satisfies the GVM condition at telecom wavelengths, therefore, the intrinsic spectral purity is much higher than that at the near-infrared wavelength range. We have achieved visibilities of over $96 \%$ in quantum correlation measurement, an $S$ value of $2.76 \pm 0.001$ in Bell's inequality measurement, and fidelities of $0.98 \pm 0.0002$ in quantum state tomography. The photons were detected by highly efficient SNSPDs and coincidence counts of $20 \mathrm{kcps}$ were achieved at $10 \mathrm{~mW}$ pump. This entangled 
photon source is compact, robust, highly-entangled, ultra-bright and spectrally highly pure. Our GVM-PPKTP-Sagnac scheme will be useful for quantum information and communication systems.

\section{Acknowledgments}

The authors are grateful to N. Matsuda, N. Singh, F. Wong and R. Ursin for helpful discussions. This work was supported by the Founding Program for World-Leading Innovative R\&D on Science and Technology (FIRST). 